\title{
The Difficulties of Congenital Syphilis Diagnosis about 3 Cases at Libreville, Gabon
}

\author{
C. M. Essomo Megnier-Mbo',2,3, S. Mayi², Y. Vierin'3, A. Ndjoyi Biguino4, \\ J. Koko3 ${ }^{3}$ A. Moussavou ${ }^{3}$ \\ ${ }^{1}$ Service de Réanimation Néonatale et Néonatologie HIAOBO, Libreville, Gabon \\ ${ }^{2}$ Departement Mère Enfant HIAOBO, Libreville, Gabon \\ ${ }^{3}$ Département de Pédiatrie, Faculté de Médecine, Libreville, Gabon \\ ${ }^{4}$ Laboratoire de Microbiologie, Faculté de Médecine, Libreville, Gabon \\ Email: owonomegniermbo@yahoo.fr
}

Received 22 February 2014; revised 20 March 2014; accepted 27 March 2014

Copyright (C) 2014 by authors and Scientific Research Publishing Inc.

This work is licensed under the Creative Commons Attribution International License (CC BY).

http://creativecommons.org/licenses/by/4.0/

(c) (i) Open Access

\section{Abstract}

First described embryo fetopathy, congenital syphilis remains a public health problem mostly in developing countries. The diagnosis mainly based on bacteriological and immunological evidence of mother-child couple is not always easy, as it is shown in our three clinical cases. Those three clinical observations demonstrate the difficulties encountered in the diagnosis of congenital syphilis in our country where only the TPHA (Treponema Pallidum Haemaglutination Assay) and VDRL (Venereal Disease Research Laboratory) tests are the only ones to be routinely carried out. Actually, these tests can be negative at the earliest stage of the syphilis or in case of zonal phenomenon. In addition, maternal antibodies could be found in child blood, even if the baby is in good health. At last, the child could have been contaminated belatedly while tests were negative at the third month of pregnancy. Congenital syphilis still exists in our developing countries and, in order to better manage this pathology, a proposition of an efficient algorithm is submitted.

\section{Keywords}

Congenital Syphilis, Diagnosis, Difficulties, Libreville, Gabon

\section{Introduction}

Congenital syphilis is a public health problem mostly in developing countries where its prevalence remains high [1]-[5]. After having practically disappeared in industrialized countries, we are witnessing a resurgence of the disease since 2001, mostly in women from marginalized populations for cultural reasons such as "gens du 
voyage” (Gypsy group) or those living in social and economic precariousness [6]-[9].

In Gabon, the presence of a remaining high number of unattended pregnancies despite of free medical care in mother-and-child healthcare centers and the high seroprevalence of the illness (13.3\%) could explain the found cases [10] [11].

The diagnosis, which relies essentially on bacteriological and immunological evidences, is not always easy as illustrated in our cases. Thus, it was done by combining together the anamnesis, the maternal history and the newborn biology (i.e., blood count, CRP, TPHA, VDRL) at birth along with skeleton's X-rays. The goal of this study was to display the difficulties inherent in that diagnosis and to propose a therapeutically management algorithm for patients.

\section{Observations}

\subsection{Case 1}

It was about a male baby born at the right term (38 weeks of amenorrhea). The pregnancy was unattended. The syphilitic serology realized at the second trimester of pregnancy had shown a positive TPHA test along with positive VDRL. The mother was not treated. The delivery took place at home. The baby cried immediately as recommended, and the newborn was transferred at the hospital for examination and medical care management. At the entry, it presented intrauterine harmonious growth retardation with a body weight of 2000 grams (gr), a size of 46 centimeters $(\mathrm{cm})$ and a cranial perimeter of 31 centimeters $(\mathrm{cm})$. The clinical examination revealed a seropurulent nasal discharge obstructing the nostrils in places, a dermatosis made of post-blistered sweating in places, located in lower limbs (from knees to ankles), back of hand, nose rim, upper lip, umbilical region along with palmo-plantar desquamation (Figure 1). We also noticed an incurvation of the four limbs, mostly pronounced in the lower ones, a hepatomegaly (HSMG) during the abdominal auscultation, and a slight decrease of

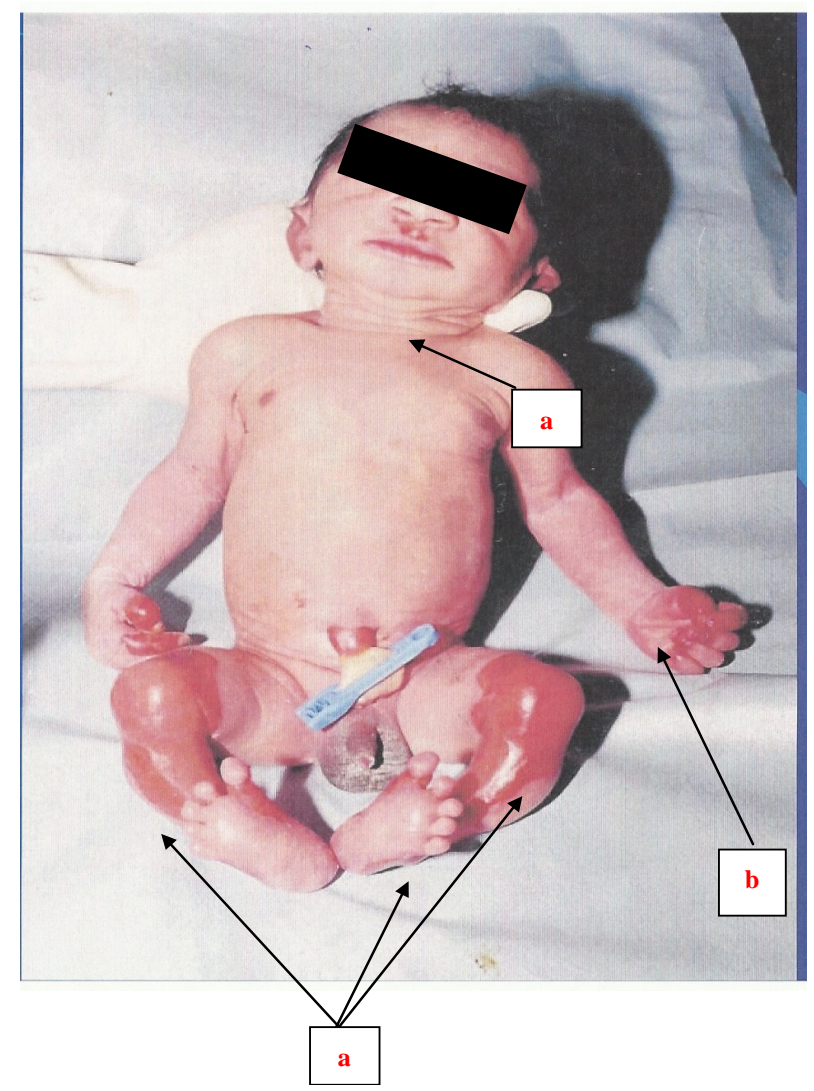

Figure 1. Illustration of a newborn suffering from cutaneomucous membrane lesions (Case 1): a) post-blistered sweating and b) Palmo-plantar desquamation. 
spontaneous motility. Cardio-pulmonary auscultation was normal along with the all the remaining tests. The newborn was hospitalized.

Facing that severe neonatal infectious overall clinical picture, triple anti-biotherapy (ampicillin-cefotaximnetilmicin) was prescribed, while awaiting bacteriological tests results.

Complementary tests results were the following: Leucocytes: 27,500 with $60 \%$ of polynuclear neutrophils; hemoglobin at $14 \mathrm{~g} / \mathrm{dL}$; platelets at $160,000 / \mathrm{mm}^{3}$; C-reactive protein (CRP) dosage at $165 \mathrm{mg} / \mathrm{L}$. Syphilitic serology of the baby was negative. Cutaneous lesions swabbing was negative especially with the absence of Treponema pallidum. Skeleton radiographs displayed metaphysary affection made of light bands predominant in lower limbs long bones, and the presence of metaphysary notches at both tibia upper-inner extremities, evocating a Wimberger positive syndrome (Figure 2).

A treatment made of Penicillin G was installed during ten days under the cover of a corticotherapy. The evolution was favorable with regression of the above-described clinical radiological signs.

\subsection{Case 2}

We dealt with a four weeks premature male newborn (34 WA). The pregnancy was unattended. No ultrasound has been performed. Syphilitic serology realized at the first trimester has shown negative TPHA and VDRL tests.

The delivery took place at home. The baby had cried immediately. APGAR score could not be determined. At birth, the baby presented harmonious intrauterine growth retardation with a body weight of 1500 gr, a size of 40 $\mathrm{cm}$ and a cranial perimeter of $29 \mathrm{~cm}$. The clinical examination at birth showed a purulent conjunctivitis, hepatic overwhelming and a discreet incurvation of the four limbs mostly pronounced in the lower ones with decrease of spontaneous motility. All the remaining clinical tests were uninfluential, with absence of cutaneo-mucous membrane lesions.

The paraclinical check-up showed white blood cells rate at 25,000 with predominant polynuclear neutrophils, red blood cells at $3.3 \times 10^{6} / \mathrm{mm}^{3}$; hemoglobin rate at $12.3 \mathrm{~g} / \mathrm{dL}$, platelets at $160,000 / \mathrm{mm}^{3}$; The C-reative protein was at $145 \mathrm{mg} / \mathrm{L}$ and the syphilitic serology was positive (for both TPHA and VDRL).

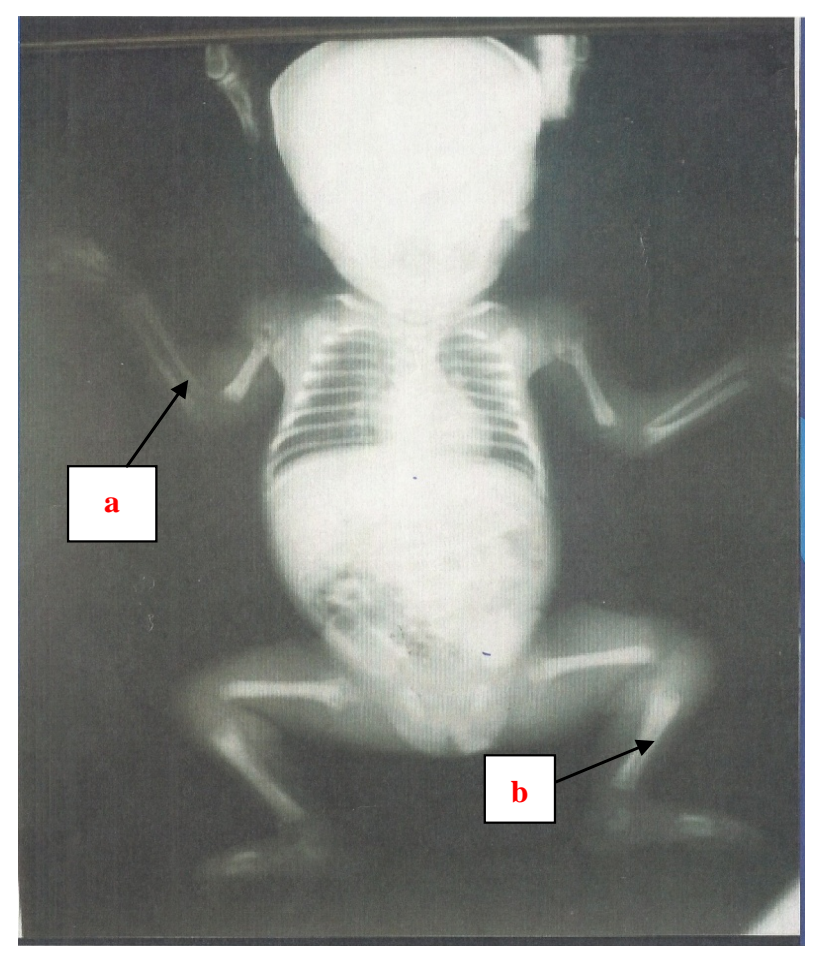

Figure 2. X-rays diagram displaying osteo-articular lesions (Case 1): The metaphysico-diaphysary affection appears easily (a and b). 
Skeleton's X-rays showed predominant metaphyso-diaphysary affection in long bones with positive Wimberger test typical of syphilitic osteochondritis (Figure 3). The newborn was placed under antibiotics treatment based on penicillin $\mathrm{G}$ at the posology of 100,000 U/kg/day during ten days. The evolution was favorable.

\subsection{Case 3}

It was about a male baby born at the right term (39 WA). The pregnancy was mismanaged. The serology performed at the last trimester was positive at the TPHA and VDRL tests. The mother was not treated. The delivery occurred in a hospital. At birth, the baby was presenting signs of hypotrophy (weight: 2100 gr; size: $44 \mathrm{~cm}$; and cranial perimeter of $31.3 \mathrm{~cm}$ ). The clinical exam at birth was normal. The baby's syphilitic serology was positive (TPHA and VDRL, without any precision of mentioned titration). The radiography of skeleton was absolutely normal along with all the biological check-up.

Therapeutically management issues had come up while facing the absence of an irrefutable diagnosis. After discussion, a treatment based on penicillin G had been recommended following the posology of 100,000 $\mathrm{U} / \mathrm{kg} /$ day during ten days. We lost the track of the newborn because the parents have checked-out without leaving no information or medical advice.

\section{Discussion}

Those three observations perfectly illustrated the difficulties encountered when it comes to deal with congenital syphilis diagnosis and came up with the problem of the therapeutically management (Table 1).

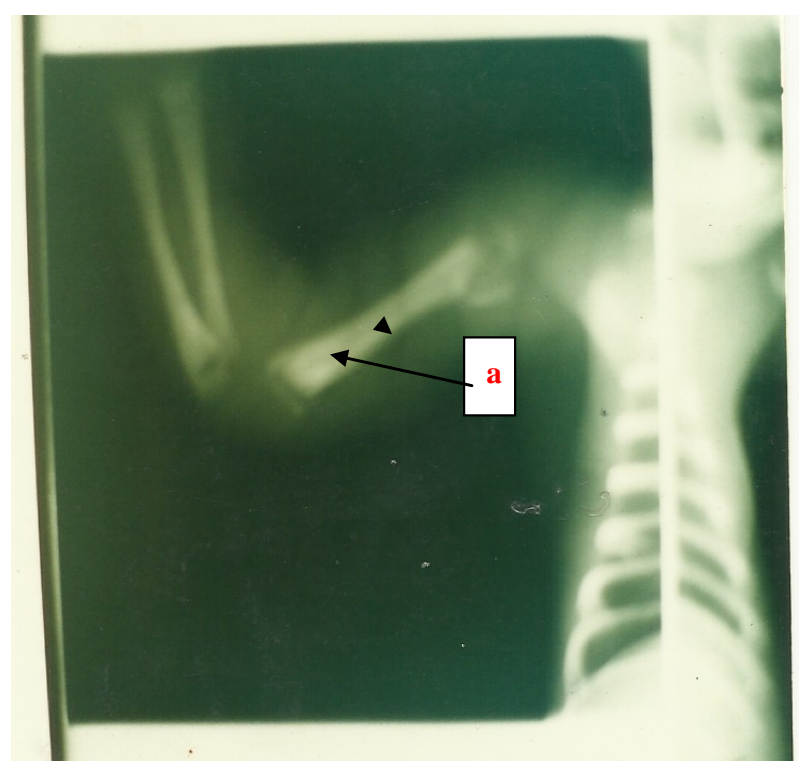

Figure 3. Right antero-brachial X-rays showing osteo-articular lesions (Case 2). The mataphysico-diapdysary affection (a) appears clearly.

Table 1. Summary of clinical information.

\begin{tabular}{lccc}
\hline & Case 1 & Case 2 & Case 3 \\
\hline Mismanaged pregnancy & + & + & + \\
Maternal syphilis serology & + & - & + \\
Newborn & RCIU & RCIU & RCIU \\
Cutaneous lesions & + & + & - \\
HSMG & + & + & - \\
Bone lesions & + & + & - \\
Newborn syphilis serology & - & Congenital syphilis? & Congenital syphilis? \\
Conclusion & Congenital syphilis? & & + \\
\hline
\end{tabular}


In fact, congenital syphilis remains a worrying form of syphilis [12]. Its increasing frequency is parallel to the recrudescence of adult primary and secondary syphilis. It is mostly evoked in cases of untreated or badly managed maternal syphilis. According to the World Health Organization (WHO), two millions of pregnant women are concerned by the illness every year, and only $17 \%$ undergo a serological check-up at the beginning of their pregnancy [13]. About 25\% of pregnancies have a pathological evolution (in utero death, spontaneous abortion...), $60 \%$ of newborns are asymptomatic at birth, and $25 \%$ of those are underweighted. The agent responsible of the disease is a spirochete, the Treponema pallidum [14].

The fetus contamination is possible at any moment of the pregnancy but mostly after the fourth month (protector effect of Langherans' cells). That mother-to-child transmission is almost ranging from $70 \%$ up to $100 \%$ if the maternal syphilis is not treated; it is about $40 \%$ if it is precociously latent ( $<1$ year), and it is about $10 \%$ if it is belatedly latent (>1 year) [15]-[17].

The diagnosis is not always easy. It is evoked while facing an untreated maternal positive syphilitic serology associated to cutaneous and bone lesions along with a hepatosplenomegaly at birth [5] [16] [18]. It can be suspected in utero by using ultrasound that displays non-specific signs such as hypotrophy, oligoamnios and other signs leading towards an infectious fetopathy such as fetal hydrops or the fetoplacental anasarca, microcephaly, hyperechogene intestine, hepatosplenomegaly and the increasing of placental thickness [15]. Table 2 displays precocious congenital syphilis clinical signs frequencies registered by Chawla in Zimbabwe in 1986 [18].

The irrefutable diagnosis is based on the finding of the treponema by using immunofluorescent or black-tinted microscopy preparations of placental or cutaneo-mucous membrane lesions along with serological tests [3] [15] [19] [20]. Placental histological affection by treponema corresponds to a villus hypertrophy, an obturating endovascular proliferation or an acute or chronic villus inflammation. Those lesions are not specific, but their association strongly suggests the diagnosis [21] [22].

However, that direct test has a low output for multiple reasons (local or general antibiotics treatment previously applied, bad smear test, inconsistent lesions) and is almost no longer used [23]. Serological tests, currently used are divided in two groups: non-treponemic tests (Venereal Disease Research Laboratory) and treponemic tests (Treponema Pallidum Haemagglutination Assay along with the Fluorescent Treponemal Assay) [24]. The VDRL uses a cardiolipidic antigen. Based on a single sampling, it does not allow reaching a conclusion between a progressive syphilis and a passive crossing of maternal antibodies. Meanwhile, a rate four-time superior to that of the mother in the newborn is likely in favor of the infection. The TPHA test uses a treponemic antigen and is more specific than the VDRL test. When they are combined, VDRL and TPHA have a good sensitivity, but still under that of immunofluorescence.

The FTA-abs-IgM is a highly sensitive method but it is costly and difficult to realize. It is based on a serological reaction between inactive treponema and specific seral IgM. The method helps to confirm the diagnosis of congenital syphilis.

\begin{tabular}{lcc}
\hline Table 2. Frequency of congenital syphilis clinical signs registered in Zimbabwe (Chawla et al., 1986). \\
\hline & Number of cases & Percentage (\%) \\
\hline Low weight at birth & 41 & 77 \\
Hepatomegaly & 28 & 53 \\
Splenomegaly & 27 & 51 \\
Hepatosplenomegaly & 26 & 49 \\
Icterus & 25 & 47 \\
Cutaneous lesions & 20 & 38 \\
Thrombopeny & 12 & 38 \\
Respiratory failure & 10 & 19 \\
Anasarca & 3 & 6 \\
Pseudo paralysis & 2 & 4 \\
Coryza & 2 & 4 \\
Anemia & 16 & 50 \\
\hline
\end{tabular}

a. Blood formula numerations could be performed only for 32 newborns. 


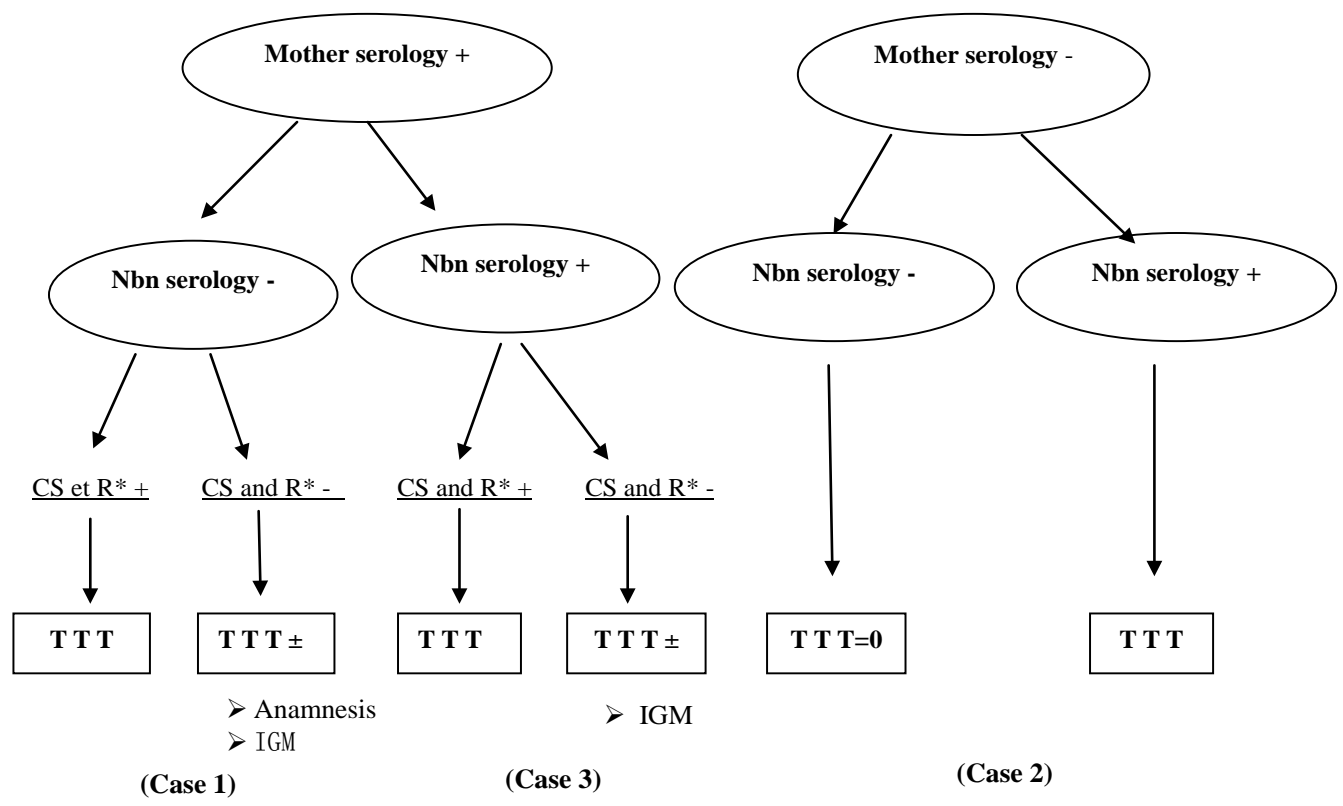

Figure 4. Congenital syphilis medical management's Algorithm. Abbreviations list: Nbn: newborn; CS: clinical signs; R*: Rx; TTT: treatment; IGM: immunoglobulins.

In addition, antibodies (IgG) could be searched in the cebrospinal fluid (CSF) by using immunofluorescence reaction (FTA-abs) or TPHA reaction. That research does not allow differentiating between central nervous system affection and simple antibodies passing if cytology and CSF chemistry are normal. Searching IgM in the CSF is possible, but it is technically difficult to realize in routine. When it is positive, it asserts central affection, but it does not elude the diagnosis.

In industrialized countries, other techniques such as the polymerase chain reaction (PCR) are realized on amniotic liquid sampling, placenta or umbilical chord, and using the solid phase hemabsorption assay (SPHA) by dosage of IgM through immunocaptation on blood and CSF sampling. The results are good.

Those three clinical observations demonstrate the difficulties of congenital syphilis diagnosis in our country wherein only the TPHA and VDRL tests are available. In fact, both tests can be negative at the precocious stage of syphilis or in case of zonal phenomenon (false negative due to excessive antibodies). In that case, it is better to dilute the serum in order to get the positive reaction (Case 1) [25]. In addition, maternal antibodies can be found in the newborn blood, even if it is uncontaminated when the mother is treated at the beginning of the pregnancy. Those antibodies are decreasing during the first year. Conversely, the child may have been contaminated lately (eighth month of pregnancy for example) while the tests were negative at the third month of pregnancy. Serological reactions may in this case be negative at birth and become positive later (Case 3) [26] [27].

In addition, congenital syphilis may be asymptomatic or paucisymptomatic in one third of case, but generally signs appear before the second month after birth (Case 2) [28]. In this case, bone lesions which are found in $80 \%$ of congenital syphilis are uplifting either alone or associated to cutaneous forms and they must rule out other congenital affections such as rubella, hyperparathyroidism, hemoglobinopathies, septicemias, neonatal leukemia, Silverman or Ambroise Tardieu syndrome (termed "Beaten children Disease") [29].

In the second clinical observation, we did the penicillin test and the clinical status along with radiological signs has improved markedly. This treatment can be combined with corticosteroids to prevent Herxheimer reaction due to the sudden lysis of treponemas. The Anglo-Saxons immediately use high doses of penicillin $\mathrm{G}$ $50,000-100,000 \mathrm{U} / \mathrm{kg}$ by intramuscular or intravenous way for 15 to 20 days [18]. Benzathine Penicillin in the dosage of 50,000 U/kg by IM injection is reserved for newborns at risk of clinical signs who are asymptomatic [1] [30] [31].

In our study, all three patients were treated with penicillin $\mathrm{G}$ at a dose of 100,000 U/kg/day or two infusions of 50,000 U/kg per day for a period of 10 days associated with corticosteroid treatment $2 \mathrm{mg} / \mathrm{kg} / \mathrm{d}$, despite the lack of diagnostic certainty in the third case. We have not used Benzathine Penicillin. The outcome was favorable in the first two cases and the third patient on treatment was lost. 
This study shows the difficulty that may arise in the diagnosis of congenital syphilis especially in our situation where we cannot do efficiently all syphilis serology. In this case, the overall clinical study, maternal history and syphilis serology of the mother and child determine the therapeutic management hence our proposed scheme that we termed congenital syphilis medical management's Algorithm (Figure 4) takes into account maternal syphilis serology, clinical status and child syphilis serology.

\section{Conclusion}

The congenital syphilis still exists in Gabon. Its diagnosis must be evoked based on irrefutable clinical and radiological signs and, if there is any subsisting doubt serology and bacteriological sampling should be performed on both mother and newborn. Thus, it is utterly important to detect maternal syphilis during pregnancy, which would allow a simultaneous treatment of both the mother and the fetus.

\section{References}

[1] Walker, D.G. and Walker, G.J. (2002) Forgotten but Not Gone: The Continuing Scourse of Congenital Syphilis. Lancet Infect Disease, 7, 433-436.

[2] Hoareau, C., Ranivoharimina, V., Chavet-Quéru, M.S., et al. (1999) Syphilis Congénitale. Mise au Point et Perspectives. Cahiers, 9, 38-45.

[3] Morshed, M.G. (2014) Current Trend on Syphilis Diagnosis: Issues and Challenges. Advances in Experimental Me- dicine and Biology, 808, 51-64.

[4] Araùjo, M.A., de Freita, S.C., de Moura, H.J., et al. (2013) Prevalence and Factors Associated with Syphilis in Parturient Woman in Northeast, Brazil. BMC Public Health, 13, 206.

[5] Gupta, R. and Vora, R.V. (2013) Congenital Syphilis, Still a Reality. Indian Journal of Sex Transm Disease, 34, 50-52.

[6] Dobson, S. (2004) Congenital Syphilis Resurgent. Advances in Experimental Medicine and Biology, 549, 35-40.

[7] Su, J.R., Berman, S.M., Davis, D., et al. (2010) Centers for Disease Control and Prevention (CDC). Congenital Syphilis-United States, 2003-2008. MMWR Morbidity and Mortality Weekly Report, 59, 413-417.

[8] Hussey, J., Mitchell, L., Hew, Y., et al. (2013) Preventing Congenital Syphilis-A Regional Audit of Syphilis in Pregnant Women Seen in Genitourinary Medecine Services. International Journal of STD AIDS, 2013, 27.

[9] Qin, J.B., Feng, T.J., Yang, T.B., et al. (2014) Risk Factors for Congenital Syphilis and Adverse Pregnancy Outcomes in Offspring of Women with Syphilis in Shenzen, China: A Prospective Nested Case-Control Study. Sex Transm Disease, 41, 13-23.

[10] Aplogan Ongotha, D.J.C. (2001) Santé de la mère et de l'enfant. Enquête démographique et de santé Gabon 2000: Direction Générale de la Statistique et des Etudes Economiques-Fonds des Nations Unis pour la Population-ORC Macro.

[11] Carles, G., Lochet, S., Youssef, M., et al. (2008) Syphilis et grossesse. Etude de 85 cas en Guyane française. Journal of Obstetrics \& Gynecology and Reproductive Biology, 37, 353-357.

[12] Simms, I. and Broulet, N. (2008) Congenital Syphilis Reemerging. Journal of Dtsch, 6, 269-272.

[13] Organisation mondiale de la santé (OMS) (2009) L’élimination mondiale de la syphilis congénitale: Raison et stratégie 2009. OMS, Genève, 48.

[14] Chaudhary, M., Kashyap, B. and Bhalla, P. (2007) Congenital Syphilis, Still a Reality in $21^{\text {st }}$ Century: A Case Report. Journal of Medical Case Reports, 1, 90.

[15] Narducci, F., Switala, I., Rajabally, R., et al. (1998) Syphilis maternelle et congénitale. Journal of Obstetrics \& Gynecology and Reproductive Biology, 27, 150-160.

[16] Stoll, B. (1994) Congenital Syphilis: Evaluation and Management of Neonates Born to Mothers with Reactive Serologic Test for Syphilis. Pediatric Infectious Disease Journal, 13, 845-853. http://dx.doi.org/10.1097/00006454-199410000-00001

[17] Gomez, G.B., Kamb, M.L., Newman, L.M., et al. (2013) Untread Maternel Syphilis and Adverse Outcomes of Pregnancy: A Systematic Review and Meta-Analysis. Bull World Health Organ, 91, 217-226. http://dx.doi.org/10.2471/BLT.12.107623

[18] Patterson, M.J. and Davies, H.D. (2011) Syphilis (Treponema pallidum). In: Kliegman, R.M., Behrman, R.E., Jenson, H.B. and Stanton, B.F., Eds., Nelson Textbook of Pediatrics, Chap. 210, 19th Edition, Saunders Elsevier, Philadelphia.

[19] Chwala, V., Bandit, P.B. and Nkrumah, F.K. (1989) Congenital Syphilis in the Newborn. Archives of Disease in Childhood, 143, 916-921.

[20] Maleville, J., Larregue, M. and Ball, M. (1991) Les divers aspects de la syphilis congénitale. Bulletin of the Exotic 
Pathology Society, 84, 609-613.

[21] Genest, D.R., Choi-Hong, S.R., Tate, J.E., et al. (1996) Diagnosis of Congenital Syphilis from Placental Examination: Comparison of Histopathology, Steiner Stain, and Polymerase Chain Reaction for Treponema pallidum DNA. Human Pathology, 27, 366-372. http://dx.doi.org/10.1016/S0046-8177(96)90110-0

[22] Walker, D.G. and Walker, G.J. (2002) Forgotten but Not Gone: The Continuing Scourge of Congenital Syphilis. The Lancet Infectious Diseases, 2, 432-436. http://dx.doi.org/10.1016/S1473-3099(02)00319-5

[23] Ray, J.G. (1995) Lues-Lues: Maternel and Fetal Considerations of Syphilis. Obstetrical \& Gynecological Survey, 50, 845-849. http://dx.doi.org/10.1097/00006254-199512000-00003

[24] Basse-Guerineau, A.L. and Assous, M.V. (2004) Syphilis: Les sérologies d’interprétation délicate. La revue du praticien, 54, 387-391.

[25] Jurado, P.D.R., Campbell, J. and Martin, P.D. (1993) Prozone Phenomenon in Secondary Syphilis. Has Its Time Arrived? Archives of Internal Medicine, 153, 2496-2498. http://dx.doi.org/10.1001/archinte.1993.00410210124014

[26] Herremans, T., Kortbeek, L. and Notermans, D.W. (2010) A Review of Diagnostic Tests for Congenital Syphilis in Newborns. European Journal of Clinical Microbiology \& Infectious Diseases, 29, 495-501. http://dx.doi.org/10.1007/s10096-010-0900-8

[27] Peterman, T.A., Newman, D.R., Davis, D. and Su, J.R. (2013) Do Women with Persistently Negative Nontreponemal Test Results Transmit Syphilis during Pregnancy? Sexually Transmitted Diseases, 40, 311-315. http://dx.doi.org/10.1097/OLQ.0b013e318285c5a7

[28] Gendrel, D., Moussavou, A., Engohan, E., et al. (1987) Syphilis congenitale paucisymptomatique et automedication pendant la grossesse. Médecine d'Afrique Noire, 34, 11-13.

[29] Ghadouane, M., Benjelloun, B.S. and Elharim-Roudies, L. (1995) L’atteinte osseuse de la syphilis congénitale précoce (à propos de 86 cas). REVUE DU RHUMATISME, 62, 457-461.

[30] Paryani, S.G., Vaughan, A.J., Crosby, M. and Lawrence, S. (1994) Treatment of Asymptomatic Congenital Syphilis: Benzathine versus Procaine Penicillin G Therapy. The Journal of Pediatrics, 125, 471-475. http://dx.doi.org/10.1016/S0022-3476(05)83300-1

[31] Lakdar, I.M., Ismaili, L. and Bouharrou, A. (2011) La syphilis congénitale révélée par une fracture spontanée. The Pan African Medical Journal, 10, 42-47. 